\title{
Comparto Paesaggio: Innovare senza perturbare
}

\author{
Virginia Genovese $^{1}$, Edvige Simone $^{1}$, and Manuela Lucia Spisso ${ }^{1}$
}

${ }^{1}$ Affiliation not available

\begin{abstract}
Le leggi che si sono succedute dal 1948 ad oggi hanno modificato e dato forza alla tutela del paesaggio, stabilendo che per paesaggio si intende la stratificazione delle azioni antropiche e naturali. Per sottolineare la complessità e l'importanza del paesaggio è stato proposto il caso studio di un impianto eolico off-shore. Attraverso la relazione paesaggistica è stato esaminato lo stato attuale del paesaggio naturale ed urbano in cui è inserito il progetto ed è stata stimata l'incidenza che tale progetto avrà sul contesto.
\end{abstract}

\section{Quadro normativo}

E' opportuno evidenziare che oggi in ambito nazionale l'elemento di riferimento è costituito dal D.lgs. del 22 gennaio $2004 \mathrm{n}^{\circ} 42{ }^{1}$ che introduce il Codice dei Beni Culturali e del Paesaggio, sulla scia dell'art. 9 della Costituzione ${ }^{2}$ secondo cui la Repubblica Italiana "tutela il paesaggio e patrimonio storico e artistico della nazione”. In particolare alla parte terza, art. dal 131 al 159, sono disciplinati i beni paesaggistici e all' art. 131 per paesaggio intende "il territorio espressivo di identità, il cui carattere deriva dall'azione di fattori naturali, umani e dalle loro interrelazioni”. Al capo IV del Codice è esplicitata la disciplina su controllo e gestione dei beni soggetti a tutela. Nell'art. 146 "viene stabilito che i titolari di immobili ed aree di interesse paesaggistico, tutelati dalla legge, (...), non possono distruggerli, né introdurvi modificazioni che rechino pregiudizio ai valori pae- 
saggistici oggetto di protezione" ed inoltre hanno "l'obbligo (...) ad astenersi dall'avviare lavori fino a quando non ne abbiano ottenuta l'autorizzazione".

Con Il DPCM del 12 dicembre $2005^{3}$ si è resa obbligatoria la redazione di una relazione paesaggistica, da corredare alla domanda di autorizzazione paesaggistica, che ne definisce finalità, criteri, contenuti e documentazione. Detta relazione deve essere articolata in due parti:

- la prima si compone dell'analisi dello stato attuale, della descrizione degli elaborati di progetto, degli elementi per la valutazione di compatibilità paesaggistica, degli elementi per la mitigazione ;

- la seconda comprende elaborati grafici quindi elaborati di analisi e di progetto ed elementi per la valutazione di compatibilità paesaggistica.

La documentazione contenuta nella domanda di autorizzazione paesaggistica indica lo stato attuale del bene paesaggistico interessato, la presenza di elementi di valore paesaggistico o beni culturali tutelati dal codice, gli elementi di mitigazione e compensazione e deve contenere e accertare la compatibilità rispetto ai valori paesaggistici riconosciuti dal vincolo, la congruità con i criteri di gestione, la coerenza con gli obiettivi di qualità paesaggistica ${ }^{4}$.

Inoltre con l'emanazione del DPR 13 febbraio $2017{ }^{5}$ sono state definite tre fattispecie: interventi soggetti ad autorizzazione paesaggistica (D.lgs 22-01-2004 art. 146); interventi non soggetti ad autorizzazione ( DPR 13-02-2017 art. 2 alleg. A); interventi soggetti ad autorizzazione paesaggistica semplificata ( DPR 13-02-2017 art3 alleg. B).

\section{Caso Studio: Parco Eolico}

Il progetto prevede la realizzazione di un parco eolico costituito da 95 aerogeneratori e copre un'area di circa $77 \mathrm{~km}^{2}$ su un perimetro di $40 \mathrm{~km}$, ad una distanza minima dalla linea di costa di $10,5 \mathrm{~km}$. Le turbine saranno grigio chiaro poiché questo colore neutro le rende particolarmen- 
te discrete all'impatto visivo ${ }^{6}$. Per la definizione del tracciato del cavidotto on-shore sono state valutate due alternative.

Come previsto dal DPCM del 12 dicembre 2005, la relazione paesaggistica ${ }^{7}$ si articola in una prima parte di analisi del contesto paesaggistico e una seconda di valutazione dell'incidenza paesaggistica. La prima fase dell'analisi del contesto consiste nell' andare ad esaminare la normativa vigente. In particolare sono due i piani che si occupano dell'argomento paesaggistico: il Piano Urbanistico Territoriale Tematico per il paesaggio e il Piano Piano Paesaggistico Territoriale Regionale. Dall'analisi della normativa risulta che per il Piano Urbanistico Territoriale Tematico per il paesaggio , considerato che il cavidotto on-shore sarà completamente interrato e che le condizioni morfologiche preesistenti saranno ripristinate, l'opera non risulta in contrasto con le indicazioni del piano ${ }^{8}$. Invece il Piano Paesaggistico Territoriale Regionale favorisce la realizzazione di impianti paragonabili a quello in progetto, mediante la mitigazione visuale, l'inserimento paesaggistico e l'opportuna localizzazione degli interventi; definisce le aree compatibili e sensibili per la localizzazione di impianti eolici di grandi dimensioni nelle quali l'area di progetto rientra; individua le Unità Paesaggistiche Elementari che ricadono nell'ambito di progetto : UPE1, UPE2 e UPE3.

Nella seconda fase del processo di analisi si verifica se l'area di progetto è sottoposta a vincoli. In questo caso l'area è interessata da aree protette, vincoli relativi alla zona litorale e al sistema di “stratificazione storica dell’organizzazione insediativa" (tratturi).

La fase seguente consiste nell'andare a individuare le caratteristiche paesaggistiche dell'area di studio. La metodologia utilizzata per la determinazione del livello di impatto paesistico consta nel considerare una correlazione tra la sensibilità del sito e l'incidenza esercitata dal progetto. Ai fini della valutazione della sensibilità paesistica per ogni Unità Paesaggistica Elementare sono state analizzate tre componenti: morfologico-strutturale, vedutistica e simbolica. La valutazione qualitativa della sensibilità paesistica ${ }^{9}$ del sito viene espressa utilizzando la classificazione di sensibilità paesistica bassa, medio-bassa, media, medio-alta, alta. 
Delle tre unità paesaggistiche considerate, l'UPE1 ha ottenuto complessivamente un giudizio Medio-alto e Medio per l'UPE2 e l'UPE3.

Successivamente sono state studiate le fasi di analisi dell'incidenza paesaggistica, di stima dell'incidenza visiva e di stima del grado di impatto paesaggistico che costituiscono la valutazione dell'incidenza paesaggistica. Quest'ultima ha lo scopo di analizzare l'impatto paesaggistico che, in questo caso, sarà determinato dall'istallazione dell'impianto off-shore.

Per quanto riguarda l'analisi dell'incidenza paesaggistica ${ }^{10}$, la valutazione del grado di incidenza è correlata alla definizione della classe di sensibilità paesistica del territorio interessato dall'opera. Ciò significa verificare se questa induca cambiamenti paesisticamente significativi. Nello specifico bisogna verificare i rapporti tra il progetto ed una serie di criteri:

- Incidenza morfologica e tipologica.

- Incidenza linguistica: Stile, materiali, colori.

- Incidenza visiva.

- Incidenza ambientale.

- Incidenza simbolica.

Data la natura dell'intervento, risulta determinante l'analisi dell'incidenza visiva, poiché un impianto ubicato in mare non è in grado di alterare la morfologia e la tipologia del territorio, né di comprometterne il simbolismo ${ }^{11}$. La valutazione qualitativa della visibilità dell'opera viene espressa utilizzando la classificazione di visibilità bassa, medio-bassa, media, medio-alta e alta.

La seconda fase interessa la stima dell'incidenza visiva delle opere. Al fine di mostrare da quali zone sarà visibile l'impianto, è stata elaborata la carta dell'intervisibilità. Per determinare con oggettività la possibile area di influenza dell'impatto visivo e la conseguente distanza di influenza, si è proceduto considerando l'angolo di visione umano, sia orizzontale che verticale. Considerando che la distanza di influenza è maggiore nel campo visivo verticale, si è deciso di riportare quest'ultima 
come distanza di riferimento per la realizzazione della carta dell'intervisibilità estendendo il bacino visuale da 17 a $35 \mathrm{~km}$ allo scopo di interessare tutte le coste del Golfo e non solo una piccola porzione.

Successivamente sono stati elaborati una serie di fotoinserimenti dai punti di vista ritenuti più significativi. Per via del colore grigio chiaro delle turbine e in presenza di particolari condizioni climatiche, come foschia, annuvolamenti, sole abbagliante, il parco potrebbe risultare meno visibile $^{12}$. Sono stati riportati i valori qualitativi dell'incidenza visiva dell'opera, con riferimento alle tre Unità Paesaggistiche Elementari considerate.

Infine viene riportata una tabella qualitativa del grado d'impatto paesaggistico che è calcolato attraverso il confronto tra la Sensibilità Paesaggistica e la Visibilità dell'Opera.

Ci siamo poi occupate di trasformare i valori qualitativi in valori quantitativi.

Risulta che il valore dell'impatto paesistico per l'UPE 1 è 12 e per l'UPE 2 e l'UPE 3 è 6.

\section{Conclusione}

Il presente studio ha avuto come obiettivo la valutazione dell'incidenza paesaggistica del Parco Eolico ${ }^{13}$. Le analisi sono state svolte attraverso la caratterizzazione del contesto paesaggistico e la successive analisi per rilevare il grado di incidenza paesaggistica, con particolare riferimento al grado di incidenza visiva. In conclusione l'impatto paesistico risulta essere sopra la soglia di rilevanza ma sotto la soglia di tolleranza, quindi è importante l'analisi degli impatti per riuscire a realizzare opere che non vadano a perturbare l'equilibrio paesaggistico. 


\section{References}

1.Bosetti \& Gatti. Decreto Legislativo 22 gennaio 2004, n. 42 Codice dei beni culturali e del paesaggio, ai sensi dell' articolo 10 della legge 6 luglio 2002, n. 137.

2.Bifulco, R. Costituzione della Repubblica Italiana : Articolo 9.

3.Bosetti \& Gatti. Decreto del presidente del Consiglio dei ministri 12 dicembre 2005 Individuazione della documentazione necessaria alla verifica della compatibilità paesaggistica degli interventi proposti, ai sensi dell' articolo 146, comma 3, del Codice dei beni culturali e del paesaggio di cui al decreto legislativo 22 gennaio 2004, n. 42.

4.Belgiorno, V., Naddeo, V. \& Zarra, T. Tecniche per la valutazione di impatto ambientale. ASTER 1, (2011).

5.Bosetti \& Gatti. Decreto del Presidente della Repubblica 13 febbraio 2017, n. 31 Regolamento recante individuazione degli interventi esclusi dall'autorizzazione paesaggistica o sottoposti a procedura autorizzatoria semplificata.

6.Scannapieco, D., Naddeo, V. \& Belgiorno, V. Sustainable power plants: A support tool for the analysis of alternatives. Land Use Policy 36, 478-484 (2014).

7.WPD. Studio di Impatto Ambientale - Allegato 7: Relazione Paesaggistica - Metadato - Valutazioni e Autorizzazioni Ambientali - VAS - VIA - AIA.

8.Nesticò, A., Elia, C. \& Naddeo, V. Sustainability of urban regeneration projects: Novel selection model based on analytic network process and zero-one goal programming. Land Use Policy 99 , $104831(2020)$.

9.Vincenzo, N., Malini, B. \& Kwang-Ho, C. Frontiers in Water-Energy-Nexus-Nature-Based Solutions, Advanced Technologies and Best Practices for Environmental Sustainability. Springer 
International Publishing 1, (2020).

10.Naddeo, V., Belgiorno, V., Zarra, T. \& Scannapieco, D. Dynamic and embedded evaluation procedure for strategic environmental assessment. Land Use Policy 31, 605-612 (2013).

11.Hasan, S. W., Liu, H., Naddeo, V., Puig, S. \& Yip, N. Y. Editorial: Environmental technologies for the sustainable development of the water and energy sectors. Water Science and Technology 81, iii-iv (2020).

12.Naddeo, V. \& Korshin, G. Water energy and waste: The great European deal for the environment. Science of The Total Environment 764, 142911 (2021).

13.Murena, A. et al.. Water-Energy Nexus: Evaluation of the Environmental Impact on the National and International Scenarios. in Frontiers in Water-Energy-Nexus-Nature-Based Solutions Advanced Technologies and Best Practices for Environmental Sustainability 33-35 (Springer International Publishing, 2019). doi:10.1007/978-3-030-13068-8. 


\section{Figure Captions}

Figure 1. Termoli, Italia (Fonte: unsplash.com)

Figure 2. Sintesi della Sensibilit@x@xtempboxaa@spacefactor Г18aГ@spacefactorPaesaggistica

Figure 3. Sintesi dell'Incidenza Visiva

Figure 4. Sintesi Qualitativa della Sensibilit@x@xtempboxaa@spacefactor $\Gamma 18 a \Gamma @$ spacefactordelPaesaggio

Figure 5. Sintesi Quantitativa dell’Impatto Paesistico 


\section{Figures}

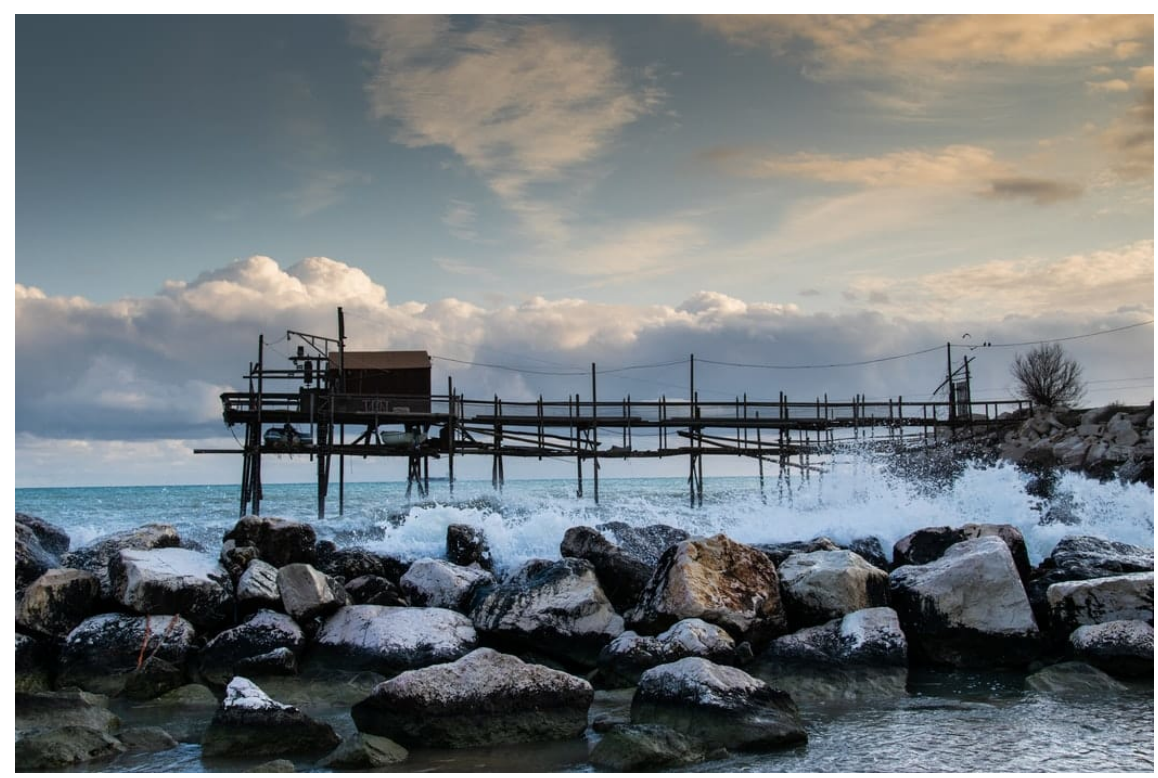

Figure 1: Termoli, Italia (Fonte: unsplash.com)

\begin{tabular}{|c|c|c|c|}
\hline & UPE 1 & UPE 2 & UPE 3 \\
\hline Morfologico-strutturale & Medio-alta & Medio-bassa & Medio-bassa \\
\hline Vedutistica & Alta & Medio & Medio \\
\hline Simbolica & Medio-alta & Medio-alta & Medio-bassa \\
\hline Valore complessivo & Medio-alta & Medio & Medio \\
\hline
\end{tabular}

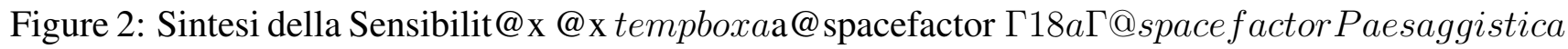

\begin{tabular}{|l|c|}
\hline Unità Paesaggistiche Elementari & Incidenza Visiva \\
\hline 1 & Media \\
\hline 2 & Medio-bassa \\
\hline 3 & Medio-bassa \\
\hline
\end{tabular}

Figure 3: Sintesi dell'Incidenza Visiva 


\begin{tabular}{|l|c|c|c|}
\hline $\begin{array}{l}\text { Unità } \\
\text { Paesaggistica }\end{array}$ & $\begin{array}{c}\text { Sensibilità } \\
\text { Paesaggistica }\end{array}$ & $\begin{array}{c}\text { Visibilità } \\
\text { dell'Opera }\end{array}$ & $\begin{array}{c}\text { Impatto } \\
\text { Paesaggistico }\end{array}$ \\
\hline 1 & Medio-alta & Media & Medio-alto \\
\hline 2 & Media & Medio-bassa & Medio \\
\hline 3 & Media & Medio-bassa & Medio \\
\hline
\end{tabular}

Figure 4: Sintesi Qualitativa della Sensibilit@x $@ \mathrm{x}$ tempboxaa@spacefactor $\Gamma 18 a \Gamma @ s p a c e f a c t o r d e l P a e s a g g i o$

\begin{tabular}{|c|c|c|c|c|c|}
\hline \multicolumn{6}{|c|}{ Impatto paesistico dei progetti = sensibilità del sito X incidenza del progetto } \\
\hline \multicolumn{7}{|c|}{$\begin{array}{c}\text { Grado di incidenza del progetto } \\
\text { sensibilità del } \\
\text { sito }\end{array}$} & 1 & 2 & 3 & 4 & 5 \\
\hline 5 & 5 & 10 & 15 & 20 & 25 \\
\hline 4 & 4 & 8 & 12 & 16 & 20 \\
\hline 3 & 3 & 6 & 9 & 12 & 15 \\
\hline 2 & 2 & 4 & 6 & 8 & 10 \\
\hline 1 & 1 & 2 & 3 & 4 & 5 \\
\hline
\end{tabular}

Figure 5: Sintesi Quantitativa dell'Impatto Paesistico 